NASA Technical Memorandum 104186
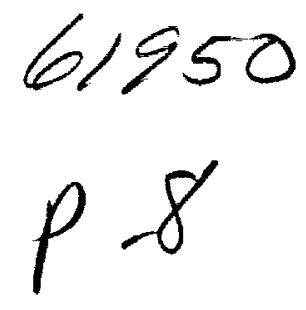

\title{
A FAST IMPLICIT UPWIND SOLUTION ALGORITHM FOR THREE-DIMENSIONAL UNSTRUCTURED DYNAMIC MESHES
}

JOHN T. BATINA

$2 \%$

DECEMBER 1991

National Aeronautics and

Space Administration

Langley Research Center

Hampton, Virginia 23665

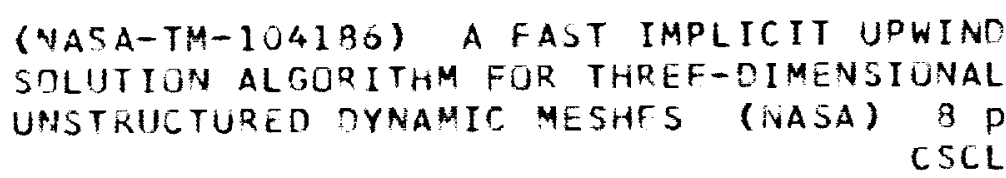

CSCL

OIA

$N 92-15050$ 
ธ....... 


\title{
A FAST IMPLICIT UPWIND SOLUTION ALGORITHM FOR THREE-DIMENSIONA! UNSTRUCTURED DYNAMIC MESHES
}

\author{
John T. Batina* \\ NASA Langley Research Center \\ Hampton, Virginia 23665-5225
}

\begin{abstract}
A fast implicit upwind algorithm for the solution of the timedependent Euler equations is presented for aerodynamic analysis involving unstructured dynamic meshes. The spatial discretization of the scheme is based on the upwind approach of Roe referred to as flux-difference splitting (FDS). The FDS approach is naturally dissipative and captures shock waves and contact discontinuities sharply. The temporal discretization of the scheme involves an implicit time-integration using a two-sweep GaussSeideI relaxation procedure. The procedure is computationally efficienl for either steady or unsteady flow problems. The paper gives a detailed description of the implicit upwind solution algorithm along with results which assess the capability. The results are presented for the NACA 0012 airfoil and for the Boeing 747 aircraft. The 747 geometry includes the fuselage, wing, horizontal and vertical tails, under-wing pylons, and flow-through engine nacelles. Euler solutions for the 747 aircraft on an unstructured tetrahedral mesh containing approximately $100,00 \times)$ cells were obtained to engineering accuracy in less than one hour $\mathrm{CPU}$ time on a Cray -2 computer.
\end{abstract}

\section{Introxluction}

In recent years signilicant progress has been made on developing numerical algorithms for the solution of the governing fluid flow equations basied on unstructured meshes. ${ }^{1-7}$ This progress includes improvements in solution accuracy as well as computational efficiency. For example, upwind methods have been developed for unstructured meshes which are based on the local wave propagation characteristics of the flow and consequently produce highly accurate solutions. ${ }^{2,3}$ Most of these upwind methods, however, use explicit time-marching schemes to integrate the governing equations in time to steady state. The explicit approach is consutationally efficient when applied to meshes that are coarse, but the rate of convergence deteriorates significantly when finer meshes are used. For cases where finer meshes are used, cither a multigrid stratlegy for convergence acceleration or an implicit temporal discretization which allows large time steps is required to obtain steady-state solutions in a computationally efficient manner. Inplicit upwind solution algorithms for unstruclured meshes in two dimensions have been reponed by the author in Ref. 8. These algorithms ane similar to the point-implicit scheme of Thareja, et al., although the methods of Ref. 8 are fully implicit and not point implicit. The purpose of the paper is to report the extension of the implicit discretization of Ref. 8 to unstructured meshes in three dimensions. This new flow solver is a fast implicit upwind algorithm for the solution of the time-dependent Fuler equations, for aero-

* Senior Research Scientist, Unsteady Aerodynamics Branch, Siructural Dynamics Division, Sconior Member AIAA dynamic analysis involving unstructured dynamic meshes. The spatial discretization of the scheme is based on the upwind approach of Roc ${ }^{10}$ referred to as flux-difference-splitting (FDS). The FDS approach is naturally dissipative and captures shock waves and contact discontinuities sharply. The temporal discretization of the scheme involves an implicit time-integration using a two-sweep Gauss-Seidel relaxation procedure. The procedure is computationally efficient for either steady or unsteady flow problems. The paper gives a detailed description of the implicit upwind solution algorithm along with results which assess the capability. The results are presented for the NACA 0012 airfoil and for the Boeing 747 aircraft.

\section{Euler Equations}

In the present study the flow is assumed to be governed by the three-dimensional time-dependent Euler equations which may be written in integral form as

$$
\frac{\partial}{\partial !} \int_{\Omega} Q d V+\int_{\partial \Omega}\left(E n_{r}+F n_{y}+C n_{z}\right) d S=0
$$

where the vector of conserved variables $Q$ and the convective fluxes $E, F$, and $(i$ are given by

$$
\begin{aligned}
& Q=\left\{\begin{array}{c}
\rho \\
\rho u \\
\rho v \\
\rho u \\
e
\end{array}\right\} \\
& E=\left\{\begin{array}{c}
\rho l \\
\rho / u+p \\
\rho(J) \\
\rho / w \\
\left.(c+p) \sigma^{\prime}+x_{u}\right)
\end{array}\right\} \\
& H=\left\{\begin{array}{c}
\mu v \\
\rho v a \\
\rho v+p \\
\rho v w \\
(c+p) V+y_{\imath} p
\end{array}\right\} \\
& G=\left\{\begin{array}{c}
\rho W \\
\rho W u \\
\rho W v \\
\rho W u+p \\
(c+p) W+z_{t} p
\end{array}\right\}
\end{aligned}
$$


The velocities $U, V$, and $W$ are defined by

$$
U=u-x_{\ell}, \quad V=v-y_{\ell}, \quad W=w-z
$$

where $x_{t}, y_{t}$, and $z_{1}$ are the grid speeds in the $x, y$, and $z$ directions, respectively, and the pressure $p$ is given by the equation of stale for a perfect gas

$$
p=(\gamma-1)\left[r-\frac{1}{2} \rho\left(u^{2}+v^{2}+w^{2}\right)\right]
$$

The above equations have been nondimensionalized by the freestream density $p_{x}$ and the freestream speed of sound $a_{x}$. Also, the second integral in Eq. (1) is a boundary integral resulting from application of the divergence theorem, and $n_{x}, n_{y}$, and $\|$; are Cartesian components of the unit normal to the boundary surface.

\section{Spatial Discretization}

The spatial discretization is based on Roe's flux-difference splituing which is herein implemented as a cell-centered scheme whereby the flow variables are stored at the centroid of each tetrahedron and the control volume is simply the tetrahedron itself. Consequently, the spatial discretization involves a flux balance where the fluxes across the four faces of a given tetrahedron are summed as

$$
\sum_{m=1}^{4} \| \Delta S=\sum_{m=1}^{1}\left(k n_{r}+\cdots n_{y}+(\operatorname{mon})_{z}\right) \Delta S
$$

where $\Delta . S$ is the area of the face. The flux vector $/ /$ is approximated by

$$
H=\frac{1}{\underline{2}}\left[U\left(Q^{+}\right)+I\left(Q^{-}\right) \cdots|1|\left(Q^{+}-Q^{-}\right)\right]
$$

where $Q^{-}$and $Q^{+}$are the state variables to the left and right of the cell face and $A$ is the flux jacobian matrix given by i) $11 / \partial Q$. Also the tilde and the absolute value sign indicate that the flux jacobian is evaluated using the so-called Roe-averaged flow variables and the absolute value of the characteristic speeds.

The left and right states $Q^{-*}$ and $Q^{+}$, are determined by upwind-biased interpolations of the primitive variables $q$. In three dimensions, for a given tetrahedron $\mathrm{j}$, for example, the upwind-biased interpolation for $f^{-}$across the common face between tetrahedra $j$ and $k$ is defined by

$$
y^{-}=y_{j}+\frac{1}{6}\left[(1-k) \Delta_{-}+(1+k) \Delta_{r}\right]
$$

where

$$
\begin{aligned}
& \Delta_{+}=\eta_{k}-\eta_{j} \\
& \Delta_{-}=\eta_{j}-\eta_{k}
\end{aligned}
$$

In Eqs. (7) and (8), $\eta_{j}$ and $/ k$ are the vectors of primitive variables at centroids $j$ and $k$, respectively, and $\%$, the vector of primitive variables at node $i$ (the node of tetrahedron $j$ opposite to the face being considered), is deternined by an inverse- distance-weighted average of the flow variables in the tetrahedra surrounding node $i$. The upwind-biased interpolation for $q^{+}$is determined similarly. Also the parameter $\alpha$ in Eq. (7) controls a family of difference schemes by appropriately weighting $\Delta_{-}$ and $\Delta_{+}$. On structured meshes it is easy to show that $\kappa=-1$ yields a fully upwind scheme, $k=0$ yields Fromm's scheme, and $\kappa=1$ yields central differencing.

On highly stretched meshes, the formula for $\Delta_{+}$is modified to be

$$
\Delta_{+}=\frac{3 a}{a+b}\left(q_{k}-q_{j}\right)
$$

where $a$ and $b$ are the distances from the midpoint of the face to the centroids of tetrahedra $j$ and $k$, respectively. This formula weights the flow variables in the interpolation formula (Eq. (7)) differently to account for the stretching of the mesh. For example, by substituting $\mathrm{Eq}$. (9) into Eq. (7) and letting $\kappa=1$

$$
q^{-}=\frac{b}{a+b} q_{j}+\frac{a}{a+b} q_{k}
$$

Furthermore, in calculations involving upwind-biased schemes, oscillations in the solution near shock waves are expected to occur. To eliminate these oscillations flux limiting is usually required. The flux limiter modifies the upwind-biased interpolations for $q^{-}$and $q^{+}$such that, for example

$$
q^{-}=q,+\frac{s}{6}\left[(1-\kappa s) \Delta_{-}+(1+\kappa s) \Delta_{+}\right]
$$

where $s$ is the flux limiter. In the present study, a continuously differentiable flux limiter was employed which is defined by

$$
s=\frac{2 \Delta_{-} \Delta_{+}+c}{\Delta_{-}^{2}+\Delta_{+}^{2}+c}
$$

where , is a very small number used to prevent division by zero in smooth regions of the flow.

\section{Temporal Discretization}

The temporal discretization is an implicit time-marching scheme involving a Gauss-Seidel relaxation procedure. The scheme is derived in general by first linearizing the flux vector II according to

$$
I^{n+1}=I^{n}+\frac{\partial \|}{\partial Q} \Delta Q
$$

where $\partial / l / \partial Q$ is the flux jacobian $A$, as discussed before, and $\Delta Q=Q^{n+1}-Q^{n}$. Linearizing both flux terms on the righthand-side of Eq. (6) using Eq. (13), and ignoring the tilde on the flux jacobian, results in

$$
\begin{gathered}
{\left[\frac{n o l}{\Delta l} I+\sum_{m=1}^{1} A^{+}\left(Q_{j}\right) \Delta S\right] \Delta Q_{j}+\sum_{m=1}^{1} A^{-}\left(Q_{m}\right) \Delta S \Delta Q_{m}} \\
=-\frac{1}{2} \sum_{m=1}^{1}\left[I I\left(Q^{+}\right)+I I\left(Q^{-}\right)-|i|\left(Q^{+}-Q^{-}\right)\right]^{n}
\end{gathered}
$$

where $l$ is the identity matrix, "vol" is the volume of the tetrahedron $j$, and $\Delta Q_{m}$ is the change in flow variables in each of the four tetrahedra adjacent to tetrahedron $j$. Also in $\mathrm{Eq}$. 
(14), $A^{+}$and $A^{-}$are forward and backward flux jacobians, respectively. For flux-difference splitting, the exact jacobian $A$ (derivative of the right-hand-side of Ey. (6) with respect to $Q$ ) is too expensive to compute and thus an approximate jacobian is normally used. This is accomplished by constructing the jacobians making use of the fact that the forward and backward jacobians should have non-negative and non-positive eigenvalues (characteristic speeds), respectively. This is accomplished by expressing altematively the jacobians using similarity transformations such that

$$
A^{+}=R \Lambda^{+} R^{-1} \quad . \quad A^{-}=R \Lambda^{-} R^{-1}
$$

where $\Lambda^{+}$and $\Lambda^{-}$are diagonal matrices whose diagonal elements are the eigenvalues $\lambda^{+}$and $\lambda^{-}$defined by

$$
\lambda^{+}=\frac{1}{2}(\lambda+|\lambda|) \quad \lambda^{-}=\frac{1}{2}(\lambda-|\lambda|)
$$

and $l f$ is the matrix whose columns are the corresponding eigenvectors.

Direct solution of the system of simultaneous equations which results from application of Eq. (14) for all tetrahedra in the mesh, requires the inversion of a large matrix with large bandwidth which is computationally expensive. Instead, a Gauss-Scidel relaxation approach is used to solve the equations whereby the summation involving $\Delta Q_{m}$ is moved to the right hand side of Eq. (14). The terms in this summation are then evaluated for a given time step using the nost recently computed values for $\triangle Q_{\ldots}$. The solution proxedure then involves only the inversion of a $5 \times 5$ matrix (represented by the terms in square brackets on the left hand side of Eq. (T4)) for each tetrahedron in the mesh. Also, although the proxedure is implemented for application on (randomly-ordered) unstructured meshes, it is not a point Gauss-Seidel procedure. The method is in fact more like line Gauss-Seidel since the list of tetrahedra that make up the unstructured mesh is re-ordered from upstream to downstream, and the solution is obtained by sweeping two times through the mesh as dictated by stability considerations. The first sweep is performed in the direction from upstrearn to downstream and the second sweep is from downstream to upstream. For purely supersonic flows the second sweep is unnecessiary.

\section{Boundary Conditions}

To impose the fow tangency houndary conditions along the surface of the vehicle, the flow yariables are sel within dummy cells that are effectively inside the geometry being considered. The velocity conponents within a dummy cell, $(1, \cdots, u)_{d,}$ are determined from the values in the cell $j$ adjacent to the surface, $(u, v, w)$. This is accomplished by first rotating the components into a coordinate system that has a coordinate direction normal to the boundary face. The sign of the velocity component in this dinetion is changed (hence imposing no flow through the face) and the three velocity components are then rotated back into the original $x, y, z$ comdinate system. After considerable algebra this yields

$$
\left\{\begin{array}{l}
u \\
v \\
w
\end{array}\right\}_{d}=\left[\begin{array}{ccc}
1-2 n_{x}^{2} & -2 n_{x} n_{y} & -2 n_{x} n_{z} \\
-2 n_{x} n_{y} & 1-2 n_{y}^{2} & -2 n_{y} n_{z} \\
-2 n_{x} n_{z} & -2 n_{y} n_{z} & 1-2 n_{x}^{2}
\end{array}\right]\left\{\begin{array}{l}
u \\
v \\
w
\end{array}\right\}_{j}
$$

where $n_{x}, n_{y}$, and $n_{z}$ are the $x, y$, and $z$ components of the unit vector that is normal to the boundary face. Also, pressure and density within the dummy cell are set equal to the values in the cell adjacent to the surface.

After application of the upwind-biased interpolation formula to determine $q^{-}$and $q^{+}$at each face, the velocity components are corrected to give a "strong" implementation of the surface boundary condition according to

$$
\begin{aligned}
& u_{\text {carrected }}=u-n_{x}\left(u n_{x}+v n_{y}+w n_{z}\right) \\
& v_{\text {corrected }}=v-n_{y}\left(u n_{x}+v n_{y}+w n_{z}\right) \\
& w_{\text {corrected }}=u-n_{z}\left(u n_{x}+v n_{y}+w n_{z}\right)
\end{aligned}
$$

In the farfield a characteristic analysis based on Riemann invariants is used to determine the values of the flow variables on the outer boundary of the grid. This analysis correctly accounts for wave propagation in the farfield which is important for rapid convergence to steady-state and serves as a "nonreflecting" boundary condition for unsteady applications.

\section{Results and Discussion}

To assess the accuracy and efficiency of the implicit upwind solution algorithm, calculations were first performed in two dimensions for the NACA 0012 airfoil. These results were obtained using the unstructured mesh shown in Fig. 1. The grid has 3300 nodes and 6466 triangles, and extends 20 chordlengths from the airfoil with a circular outer boundary. Also there are 110 points that lie on the airfoil surface. Steady-state calculations were performed for the airfoil at a freestream Mach number of $M t_{x}=0.8$ and an angle of attack of $\alpha=1.25^{\circ}$. The results were obtained using both the implicit relaxation time-marching scheme and an explicit three-stage Runge-Kutta time-marching scheme. The explicit results were oblained using a CFL number of 4.0 , with residual smoothing and local time-stepping to accelerate convergence to steady state. The implicit results were obtained using a CFL number of infinity. Such a large value was used for the implicit results since the relaxation scheme has maximum damping and hence fastest convergence for very large time steps. This is in contrast with implicit approximate factorization schemes which have maximum damping for CFL numbers on the order of 10 . Also, the flux jacobians of the implicit scheme were updated only every twenty iterations.

A comparison of the convergence histories between explicit and implicit lime-marching is shown in Fig. 2(a). The "error" in the solution was taken to be the $L_{2}$ norm of the density residual. As shown in Fig. 2(a), the explicit solution is slower to converge than the implicit solution. The explicit solution takes approximately $739 \mathrm{CPU}$ secs. (2,682 iterations) on a Cray-2 computer to converge to engineering accuracy, which is taken to be a three order of magnitude reduction in solution error. In 


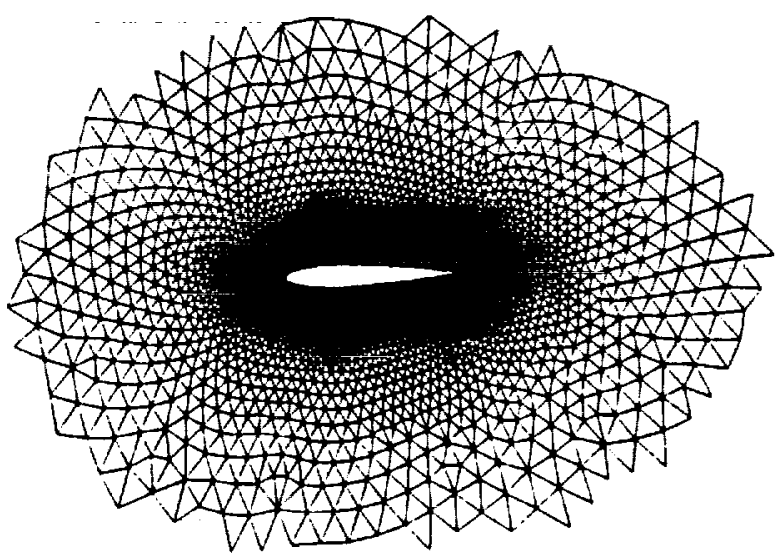

Fig. 1 Partial view of unstructured mesh of triangles about the NACA 0012 airfoil.

constrast, the implicit solution is converged to three orders of magnitude in only approximately 362 secs. (1,251 iterations). The resulting steady pressure distribution is shown in Fig. 2(b). For this case there is a relatively strong shock wave on the upper surface of the airfoil near $62 \%$ chord and a relatively weak shock wave on the lower surface near $30 \%$ chord. The pressure distribution indicates that there is only one grid point within the shock structure, on either the upper or lower surface of the airfoil, due to the sharp shock capturing ability of the Roe solver.

To assess the efficiency of the implicit upwind solution algorithm in three dimensions, calculations were performed for the Boxing 747 aircraft. These results werc obtained using the unstruclured mesh shown in Fig. 3. The 747 geomelry includes the fuselage, the wing, horizontal and vertical tails, under-wing pylons, and flow-through engine nacelles. The unstructured

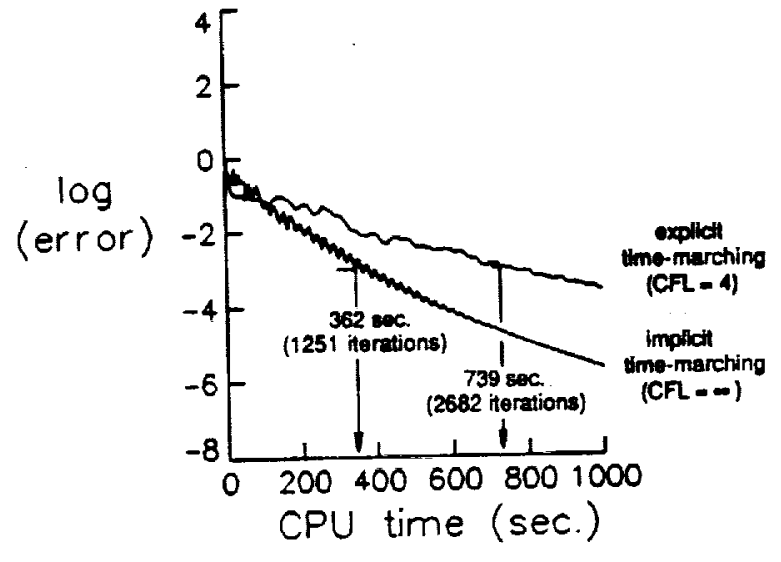

(a) convergence histories.

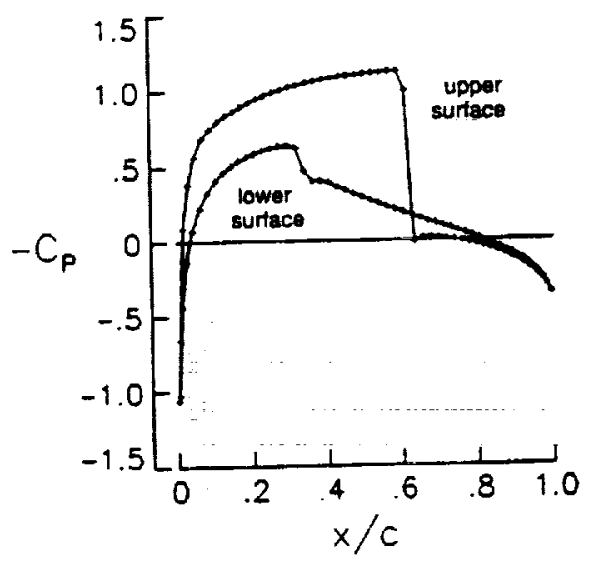

(b) pressure distribution.

Fig. 2 Comparison of steady-state results for the NACA 0012 airfoil at $M_{x}=0.8$ and $a=1.25^{\circ}$.

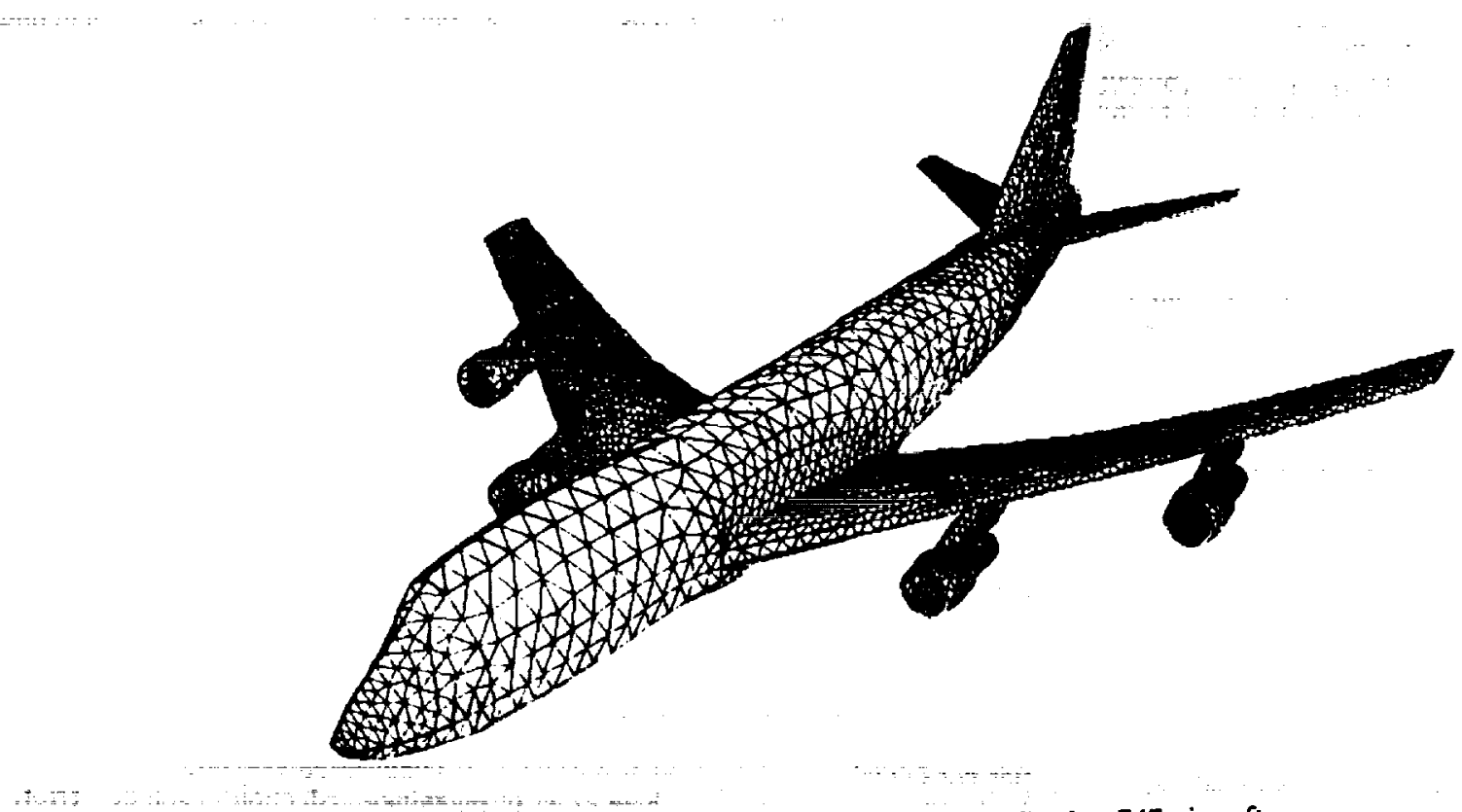

Fig. 3 Surface mesh of triangles for the Boeing 747 aircraft. 


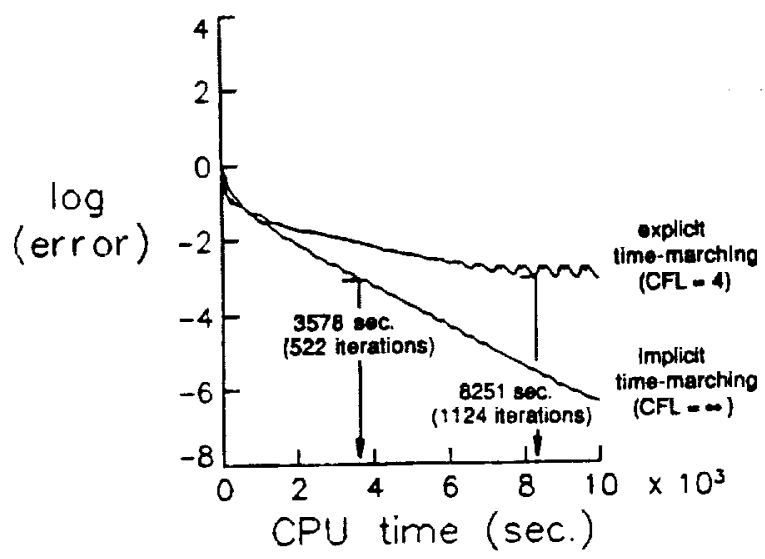

Fig. 4 Comparison of convergence histories for the Boeing 747 aircraft at $M_{x}=0.84$ and $\alpha=2.73^{\circ}$.

mesh for the 747 contains 101,475 ketrahedra and 19,055 nodes for the half-span airplane. Also there are 4,159 nodes and 8,330 triangles on the boundaries of the mesh which include the airplane, the symmetry plane, and the farfield. Sieady-state calculations were performed for the aircraft at $M_{x}=0.84$ and $n=2.73^{\circ}$. The results were obtained using both the implicit and explicit time-nuarching schenes. Similar to the NACA (0012 cases, the explicit results were obtained for the 747 using a CFL number of 4.0 , with residual smoothing and local timestepping to accelerate convergence to steady state. The implicit results were obtained using a CFL number of infinity and the flux jacobians were updated only every twenty iterations.

A comparison of the convergence histories between explicit and implicit time-marching is shown in Fig. 4. The explicit solution required 8,251 CPU secs. (1,124 iterations) on a Cray2 computer to converge the solution three orders of magnitude, whereas the implicit solution required less than half of that, or 3,578 secs. (522 steps). The resulting steady pressure coefficient contours on the surface of the 747 aircraft are shown in Fig. 5. The contours indicate that there is a significant amount of flow compression on the nose of the aircraft, along the inboard leading edge of the wing, and inside the cowl of the engine nacelle. There is flow expansion on the forward fuselage, on the horizontal and vertical tail surfaces, and on the upper surface of the wing terminated by a shock wave. Additional details of the mesh and pressure contours on the outboard pylon and engine nacelle are shown in Fig. 6. These contours show further flow expansion on the outside of the cowl and within the inner core of the engine.

\section{Concluding Remarks}

A fast implicit upwind algorithm for the solution of the time-dependent Euler equations was presented for aerodynamic analysis involving unstructured dynamic meshes. The spatial discretization of the scheme is based on the upwind approach of Roe referred to as flux-difference splitting (FDS). The FDS approach is naturally dissipative and captures shock waves and contact discontinuities sharply. The temporal discretization of the scheme involves an implicit time-integration using a twosweep Gauss-Seidel relaxation procedure. The procedure is computationally efficient for either steady or unsteady flow problems. Results were presented for the NACA 0012 airfoil and for the Boeing 747 aircraft. The 747 geometry included the fuselage, wing, horizontal and vertical tails, under-wing pylons, and flow-through engine nacelles. Euler solutions for the 747 aircraft on an unstructured tetrahedral mesh containing approxinately 100,000 cells were obtained to engineering accuracy in less than one hour CPU time on a Cray- 2 computer.

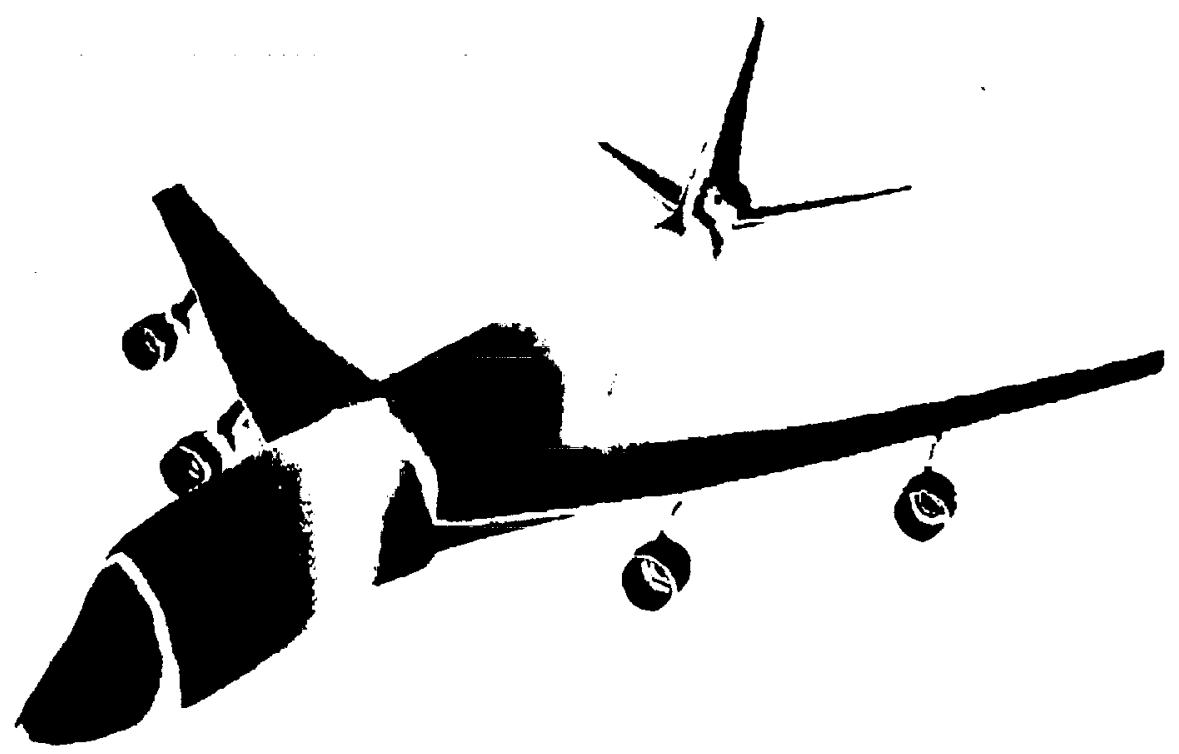

Fig. 5 Steady pressure coefficient contours on the Boeing 747 aircraft at $M_{x}=0.84$ and $a=2.73^{\circ}$. 


\section{Acknowledgements}

The author acknowledges Paresh Parikh of ViGYAN Inc. Hampton, Virginia, and Rainald Lohner of the George Washington University, Washington, D.C., for providing the advancing front method grid generation program that was used to generate the mesh for the NACA (X)12 airfoil in the present study. The author further acknowledges Neal Frink of the Transonic Aerodynamics Branch, NASA Langley Research Center, Ilampion, Virginia, for providing the unstructured tetrahedral mesh for the Boeing 747 aircraft.

\section{References}

'Salas, M. D., (editor): Accuracy of Unstructured Grid Techniques Workshop, NASA langley Restarch Cener, Hampton. Virginia, January 1990, NASA Conference Proceedings in progress.

${ }^{2}$ Batina, J. T.: Three-Dimensional Flux-Split Euler Schemes Involving Unstructured Dynamic Meshes, AIAA Paper No. 90-1649. June 1900.

${ }^{3}$ Frink, N. T.; Parikh, P.; and Pirzadeh, S.: A Fast Upwind Solver for the Euler Equations on Three-Dimensionall Unstructured Meshes, AIAA Paper No. 91-0102, January 1991 .

${ }^{4}$ Lohner, R.; and Baum, J. D.: Numerical Simulation of Shock Interaction with Complex Geometry Three-Dimensional
Structures Using a New Adaptive H-Refinement Scheme on Unstructured Grids, AIAA Paper No. 90-0700, January 1990.

${ }^{5}$ Thareja, R. R.; Morgan, K.; Peraire, J.; and Peiro, J.: A Three-Dimensional Upwind Finite Element Point Implicit Unstructured Grid Euler Solver, AJAA Paper No. 89-0658, January 1989.

${ }^{6}$ Mavriplis, D. J.: Algebraic Turbulence Modeling for Unstructured and Adaptive Meshes, AIAA Paper No. 90-1653, June 1990.

${ }^{7}$ Barth, T. J.: Numerical Aspects of Computing Viscous High Reynolds Number Flows on Unstructured Meshes, AIAA Paper No. 91-0721, January 1991.

${ }^{8}$ Batina, J. T.: Implicit Flux Split Euler Schemes for Unsteady Aerodynamic Analysis Involving Unstnuctured Dynamic Meshes, AIAA Paper No. 90-0936, April 1990.

${ }^{9}$ Thareja, R. R.; Stewart, J. R.; Hassan, O.; Morgan, K.; and Peraire, J.: A Point Implicit Unstructured Grid Solver for the Euler and Navier-Stokes Equations, AIAA Paper No. $88 \cdot(0036$, January 1988.

${ }^{\prime \prime} R(x$, P. L.: Approximate Riemann Solvers, Parameter Vectors, and Difference Schemes, Journal of Computational Physics, vol. 43, 1981, pp. 357-372.

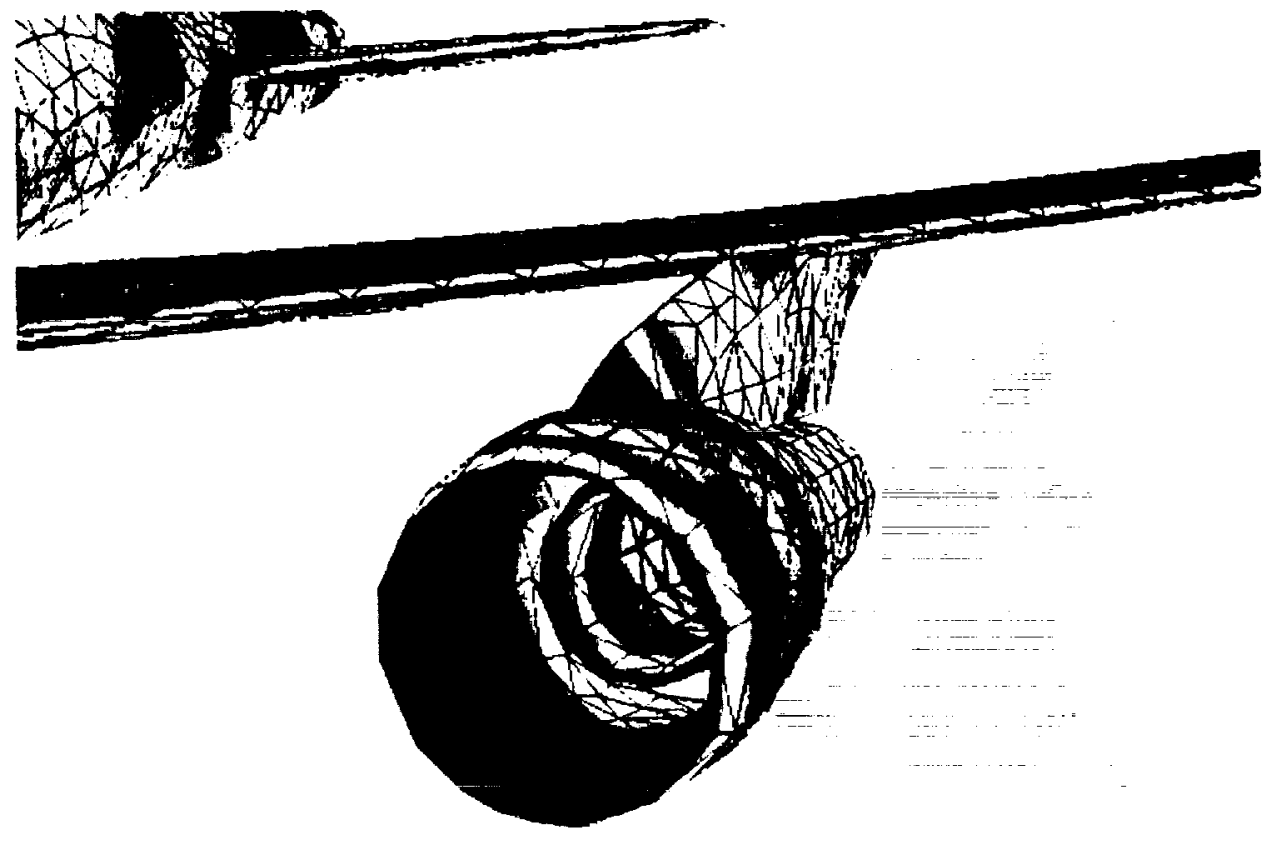

Fig. 6 Details of the surface mesh and șteady pressure coefficient contours on the outboard pylon and nacelle of the Bocing 747 aircraft at $N_{\mathrm{r}}=0.84$ and $\mathrm{r}=2.73^{\circ}$. 


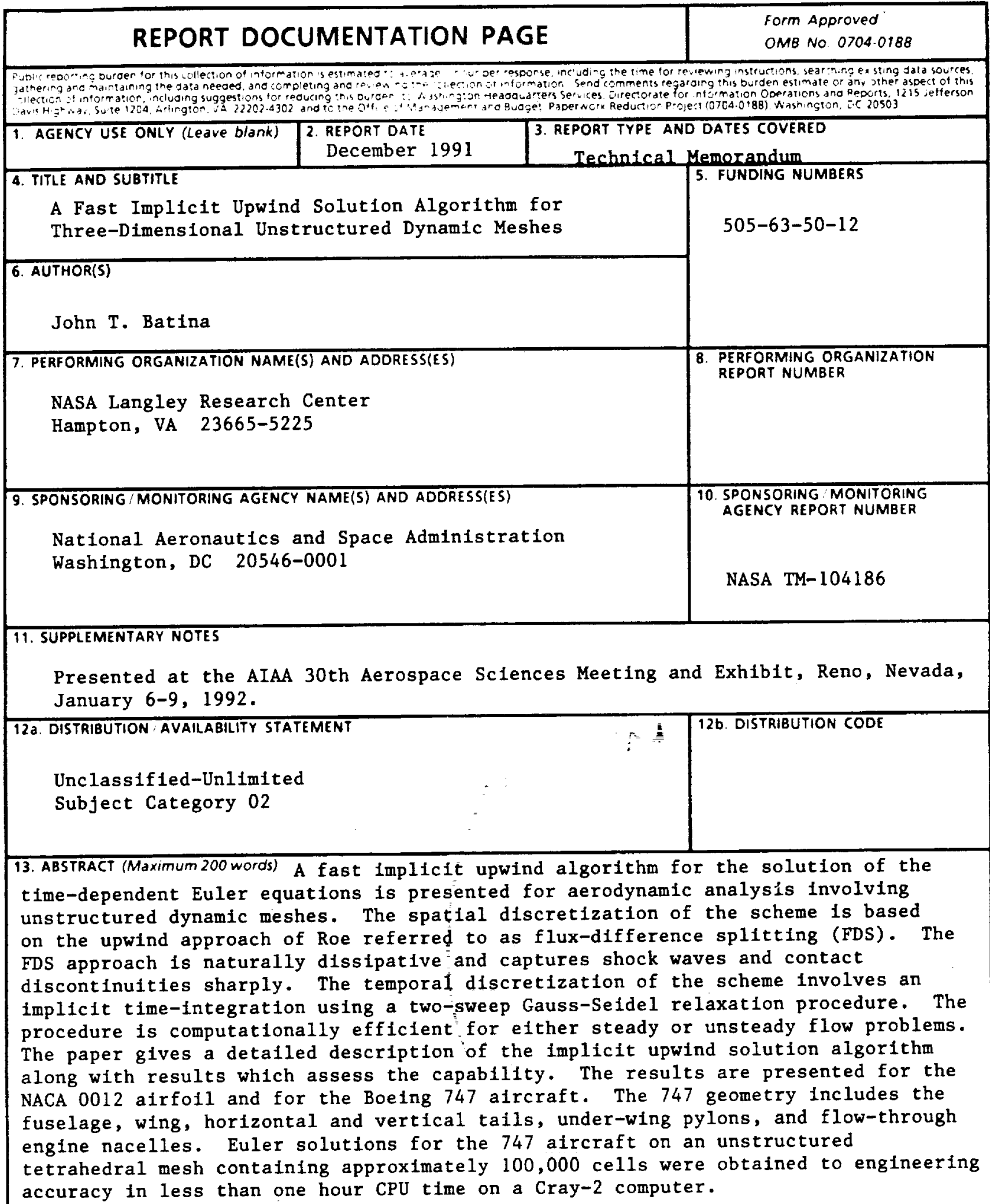

\begin{tabular}{|c|c|c|c|}
\hline \multicolumn{3}{|l|}{ 14. SUBJECT TERMS } & 15. NUMBER OF PAGES \\
\hline \multicolumn{2}{|c|}{$\begin{array}{l}\text { Computational Fluld Dynamics } \\
\text { Aerodynamics }\end{array}$} & $\begin{array}{l}\text { Aeroelasticity } \\
\text { Unsteady Aerodynamics }\end{array}$ & $\frac{7}{16 . \text { PRICE CODE }}$ \\
\hline $\begin{array}{l}\text { 17. SECURITY CLASSIFICATION } \\
\text { OF REPORT }\end{array}$ & $\begin{array}{l}\text { 18. SECURITY CLASSIFICATION } \\
\text { OF THIS PAGE }\end{array}$ & $\begin{array}{l}\text { 19. SECURITY CLASSIFICATION } \\
\text { OF ABSTRACT }\end{array}$ & 20. LIMITATION OF ABSTRACT \\
\hline Unclassified & Unclasstfied & Unclassifled & \\
\hline
\end{tabular}


